\title{
The liver in critical illness
}

\section{Felicity Hawker}

\section{Introduction}

The liver is in some ways the forgotten organ in intensive care practice. Very many more laboratory and clinical studies have investigated the role, function, and support of the lung, heart, brain, and kidney in critical illness than have studied the liver. Nevertheless, in the time of the Greek scholars, there was already acknowledgement of the role of the liver in non-hepatic diseases such as systemic sepsis, and an understanding that such involvement confers a poorer prognosis - hence the inclusion of the wisdom of Hippocrates in this compilation of classic papers. In the review article by Matuschak and Rinaldo, the reasons why liver dysfunction is associated with a poorer outcome in critical illness are explored, and the concept of the liver being a 'driving force' in multiple organ dysfunction is developed. In addition, jaundice without significant liver dysfunction is associated with left ventricular dysfunction, at least in the dog model developed by Professor Otto Better and his colleagues in Israel. This observation is relevant to the progressive resistance to inotropic and vasopressor agents in jaundiced critically ill patients.

One of the most devastating insults that can occur in critical illness is acute liver ischemia, resulting in the clinical syndrome of ischemic or hypoxic hepatitis. Although relatively common, this syndrome has been difficult to study prospectively. Consequently, the paper by Jean Henrion from Belgium offers new insights into the hemodynamic associations of ischemic hepatitis, which is, of course, a circulatory disease, not a liver disease.

Commonly used 'liver function tests' assess damage to the liver rather than its function. An understanding of the complex relationship between critical illness and liver dysfunction has been hampered by the absence of a simple test that assesses at least one of the many functions of the liver. The MEGX test, developed by Oellerich and co-workers in Germany, allows dynamic assessment of liver function, and may prove useful in the evaluation of therapies to support liver function in critical illness.

The complex issues involved in the management of patients with acute liver failure exemplify the many important functions of the healthy liver. With the advent of liver transplantation as the principle treatment for patients with fulminant hepatic failure, these patients are almost always managed in ICUs servicing liver transplant units. Consequently, it is extremely important that intensive care practitioners in non-liver units understand the need to refer patients to transplant units, as well as the role of transplantation in acute liver failure and its indications. The seminal paper by O'Grady and the group from King's College Hospital, London, develops criteria for liver transplantation in acute liver failure. That a successful outcome is possible after emergency liver transplantation for acute liver failure is shown in the early series of cases reported from the French group from Clichy and Villejuif. When transplantation is not indicated, or while awaiting a donor, the principal treatment is supportive. The paper by Harrison and co-workers, again from the King's Liver Unit, investigates the effects of n-acetylcysteine in patients with acute liver failure, and the findings of this study have resulted in widespread use of this agent in this setting. Currently, there is still no safe, effective means of artificial liver support for patients awaiting transplantation or liver regeneration. The case report from Saunders and co-workers from Cape Town, South Africa, describes an early attempt to develop such support.

Finally, patients with cirrhosis and other forms of chronic liver disease present different management challenges for the intensive care specialist. The randomized controlled trial from 
the Barcelona group shows that in patients with spontaneous bacterial peritonitis and cirrhosis, the outcome is improved by infusing albumin in addition to antibiotic therapy.

There are many classic papers that have not been included in this small selection. The majority of papers have been chosen for the underlying ideas and variety rather than for scientific rigor. They are aimed at clinical intensive care specialists with enquiring minds who work in general intensive care units, rather than experts in liver units or intensive care units servicing liver transplant units. I have found the liver a fascinating organ to study. I trust this selection of papers will likewise stimulate your interest. 
Title

\section{Whenever in fevers jaundice supervenes before seven days, this is bad}

Author

Hippocrates

\section{Reference}

Aphorism IV 60. In: Stephanus of Athens. Commentary on Hippocrates' Aphorisms, Sections III-IV. Text and translation by Westerink LG. Berlin: Akadamie Verlag, 1992

\section{Abstract}

Not applicable

\section{Summary}

Hippocrates examines a number of questions with respect to jaundice associated with systemic infection. He concludes that jaundice is a result of impairment of the separative faculty of the liver; that is, impairment of its ability to purify all the humors. He further states that this may occur as a result of obstruction of the biliary vessels or of seething inflammations, in which case the blood is distributed through the whole body with a strong admixture of bile.

\section{Citation count Not applicable}

\section{Related references}

1. te Boekhorst T, Urlus M, Doesburg W, Yap SH, Goris RJ. Etiologic factors of jaundice in severely ill patients. A retrospective study in patients admitted to an intensive care unit with severe trauma or with septic intra-abdominal complications following surgery and without evidence of bile duct obstruction. J Hepatol 1988; 7: 111-117.

2. Gimson AES. Hepatic dysfunction during bacterial sepsis. Intensive Care Med 1987; 13: $162-166$.

3. Zimmerman H, Fang M, Utili R, Seeff L, Hoofnagle J. Jaundice due to bacterial infection. Gastroenterology 1979; 77: 362-374.

\section{Key message}

Jaundice may develop in primarily non-hepatic systemic illnesses, particularly infection. In this setting, jaundiced patients have a poorer outcome.

\section{Why it's important}

This may be the first description of jaundice associated with sepsis, and its adverse effect on outcome.

\section{Strengths}

Originality. 


\section{Weaknesses}

Not evidence-based!!!

\section{Relevance}

A reminder that clinical experience and wisdom have been, and always will be, key factors in the practice of intensive care medicine 
Title

\section{Organ interactions in the adult respiratory distress syndrome during sepsis: role of the liver in host defence}

Author

Matuschak GM, Rinaldo JE

Reference

Chest 1988; 94: 400-406

Abstract

Not available

\section{Summary}

This review article explores the role of the liver in the pathogenesis of the adult respiratory distress syndrome (ARDS) and progressive multiple systems organ failure. It is argued that the normal liver plays a pivotal role in aspects of host defence that are crucial to the protection of the lung and other organs in systemic sepsis. The authors note that the fixed hepatic macrophages, or Kupffer cells, constitute nearly $90 \%$ of the body's reticuloendothelial cell mass. They further note that these cells are the major mechanism for clearance of systemic endotoxin and bacterial products. The strategic location of the liver, immediately downstream from the large gastrointestinal reservoir of bacteria and bacterial products, normally constitutes an effective first line in host control of systemic endotoxemia and bacteremia.

However, the balance may be altered by an excess load of bacterial products in the gut, mesenteric or hepatic ischemia, and Kupffer cell dysfunction, so that the phagocytic capacity of Kupffer cells is exceeded, resulting in systemic spread of bacterial products. Moreover, the authors describe the activation of Kupffer cells by the phagocytic burst, resulting in the release of an array of inflammatory mediators that are 'exported' systemically, and result in acute pathophysiological reactions in distant organs. These inflammatory mediators also have effects on adjoining hepatic parenchymal cells. Evidence is presented that the resulting hepatocyte damage may result in decreased clearance of some of these mediators, resulting in heightened and prolonged effects, and consequent amplification of multiple organ damage. Furthermore, these inflammatory mediators - particularly interleukin-1 - induce changes in hepatic protein synthesis, characterized principally by the 'acute phase response'.

Thus, this review acknowledges the role of the liver in host defence but also proposes ways in which liver dysfunction might fuel multiple organ dysfunction - particularly ARDS.

\section{Citation count 95}

\section{Related references}

1. Jarrar D, Chaudry $\mathrm{IH}$, Wang P. Organ dysfunction following hemorrhage and sepsis: mechanisms and therapeutic approaches (Review). Int J Mol Med 1999; 4: 575-583.

2. Carrico CJ, Meakins JL, Marshall JC, Fry D, Maier RV. Multiple-Organ-Failure Syndrome: the gastrointestinal tract - the motor of MOF. Arch Surg 1986; 121: 197-201.

3. Marshall JC, Lee C, Meakins JL, Michel RP, Christou NV. Kupffer cell modulation of the systemic immune response. Arch Surg 1987; 122:191-196. 


\section{Key message}

The liver plays a major role in host defence, but also has the capacity to augment the host response, with consequent adverse effects on other organs in some circumstances. This has led to the concept of the liver as 'the motor of multiple organ failure'.

\section{Why it's important}

This review was among the first to provide a plausible concept of organ system interactions that may result in ARDS and multiple organ failure in sepsis. Although some of the detail has been refined, the general principles remain accepted 15 years later.

\section{Strengths}

This paper synthesizes experimental findings from a number of disciplines - intensive care medicine, hepatology, immunology, and thoracic medicine - to arrive at a concept that was new at the time of publication, and which continues to be supported in the main by more recent evidence.

\section{Weaknesses}

This paper is not a systematic review.

\section{Relevance}

The general concepts in this review remain valid, and have led to a more concentrated focus on the gut and liver in the critically ill patient, both experimentally and in the clinical setting. Although there have been major advances in understanding of relevant factors such as splanchnic blood flow, no 'liver-orientated therapy' has yet been shown to be of value in ARDS and multiple organ failure, and the treatment of liver dysfunction remains supportive. 
Title

\section{The "jaundiced heart": a possible explanation for postopera- tive shock in obstructive jaundice}

Author

Green J, Beyar R, Sideman S, Mordechovitz D, Better O

\section{Reference}

Surgery 1986; 100: 14-19

\section{Abstract}

Patients with obstructive jaundice are susceptible to postoperative shock and kidney failure. The cause of these potentially fatal complications has not been fully clarified. The present study was designed to assess the role of myocardial dysfunction in the hemodynamic disturbance of obstructive jaundice. We studied the effect of isolated cholemia on left ventricular performance in five conscious dogs, before and 2 weeks after choledochocaval anastomosis, by using measurements of systolic time intervals (STIs) and maximal dp/dt. Mean left ventricular ejection time (LVET) decreased after cholemia from 159 $+/-2.8 \mathrm{msec}$ to $139+/-2.6 \mathrm{msec}(\mathrm{p}<0.005)$, while mean preejection period (PEP) and mean PEP/LVET were increased from $41+/-8.5 \mathrm{msec}$ to $87+/-14 \mathrm{msec}(p<0.05)$ and from $0.39+/-0.06$ to $0.62+/-0.1(p<0.01)$, respectively. During cholemia, STIs were unchanged after intravenous administration of ouabain, whereas in the control period, there was shortening of mean PEP from $71+/-8.8 \mathrm{msec}$ to $58+/-7.6 \mathrm{msec}(p<0.05)$, and of Q-S2 from $257+/-12$ msec to $235+/-14$ msec $(p<0.005)$ in response to ouabain. Maximal dp/dt decreased after choledochocaval anastomosis from $4543+/-593 \mathrm{mmHg} /$ $\mathrm{sec}$ to $3666+/-648 \mathrm{mmHg} / \mathrm{sec}(\mathrm{p}<0.025)$. We conclude that cholemia in the dog is clearly associated with impaired left ventricular performance. The present data also support a previously published in vitro study from our laboratory showing that cholemia blunts the myocardial contractile response to sympathomimetic agents. The cardiodepressor effect of cholemia may explain the increased tendency of patients with obstructive jaundice to postoperative shock and renal failure.

\section{Summary}

The effect of deep jaundice on left ventricular function was studied in five dogs. Each dog was studied before and 2 weeks after choledochocaval anastomosis (CDCA), thus each dog served as its own control. CDCA results in severe jaundice with minimal liver damage. Mean arterial blood pressure, end-diastolic left ventricular pressure, and maximal rate of increase in left ventricular pressure $(\mathrm{dp} / \mathrm{dt})$ were obtained by retrograde catheterization of the aorta and left ventricle. Systolic time intervals (pre-ejection period (PEP) and left ventricular ejection time (LVET)) were measured with simultaneous ECG and aortic pulse tracings. Systolic time intervals were measured before and after intravenous injection of ouabain.

The findings revealed that deep jaundice was associated with impaired left ventricular performance. There was a decrease in $\mathrm{dp} / \mathrm{dt}$ without changes to arterial pressure, heart rate, or preload. Moreover, there was an associated increase in mean left ventricular PEP, and a decrease in LVET when compared with predicted values. Although administration of ouabain in normal dogs (i.e. pre-CDCA) was associated with decreased PEP, no effect was observed in deeply jaundiced animals. 


\section{Related references}

1. Wakim KG, Essex HE, Mann FC. The effects of whole bile and bile salts on the perfused heart. Am Heart J 1939; 18: 171-175.

2. Bashour TT, Antonini C, Fisher J. Severe sinus node dysfunction in obstructive jaundice. Ann Intern Med 1985; 103: 384-385.

3. Binah O, Bomzon A, Blendis I, Mordohovich D, Better O. Obstructive jaundice blunts myocardial contractile response to isoprenaline in the dog: a clue to the susceptibility of jaundiced patients to shock? Clin Sci 1985; 69: 647-653.

\section{Key message}

Deep jaundice may have hemodynamic consequences even when liver function is relatively intact. It is associated with impaired left ventricular performance that is refractory to cardiac glycosides in this animal model.

\section{Why it's important}

The hemodynamic manifestations of advanced liver failure with jaundice are well described, and are basically a 'high output-low resistance' state. This paper suggests that jaundice has its own effects on cardiac function independent of liver failure.

\section{Strengths}

The authors are clearly original thinkers who have devised an animal model to shed light on a clinical problem. The model is relevant to patients with jaundice associated with multiple organ failure, as well as obstructive jaundice

\section{Weaknesses}

This is a small animal study. The methods of assessment of left ventricular function are probably valid, but are not conventional.

\section{Relevance}

Patients with multiple organ failure are frequently jaundiced, and in this setting, plasma bilirubin concentrations may be grossly elevated. Such patients often also have severe hemodynamic compromise that can be refractory to inotropic agents. Although this paper does not offer a solution to this problem, it does propose a plausible mechanism. 
Title

\section{Hypoxic hepatitis in patients with cardiac failure: incidence in a coronary care unit and measurement of hepatic blood flow}

Author

Henrion J, Descamps O, Luwaert R, Schapira M, Parfonry A, Heller F

Reference

J Hepatol 1994; 21: 696-703

Abstract

The incidence of hypoxic hepatitis was prospectively studied for 1 year in a group of high-risk patients suffering from low cardiac output in a coronary care unit. Hypoxic hepatitis, defined as an increase in serum aminotransferase activity of at least 20 times the upper limit of normal without any other cause for hepatic necrosis, was observed in 20 patients. This represents $2.6 \%$ of the 766 patients admitted to the unit during this period, and $21.9 \%$ of the 91 patients suffering from low cardiac output. Clinical, biological, and hemodynamic data were compared between 20 patients with low cardiac output and hypoxic hepatitis, and 48 patients with low cardiac output but without hypoxic hepatitis who survived more than 24 hour. In these two groups of patients, hepatic blood flow was measured by galactose clearance at low concentration. Patients with hypoxic hepatitis exhibited a higher central venous pressure $(90 \%$ versus $38 \%, p<0.001)$, as well as a lower hepatic blood flow $(867+/-377 \mathrm{ml} / \mathrm{min}$ versus $1429+/-644 \mathrm{ml} / \mathrm{min}, \mathrm{p}=0.001)$. In conclusion, although it is considered a rare hepatic disorder, hypoxic hepatitis is frequent in patients with low cardiac output admitted to the coronary care unit, and is associated with a decrease in hepatic blood flow, and passive hepatic venous congestion.

\section{Summary}

Over a 1-year period in a Belgian coronary care unit, 91 patients were identified as having a low cardiac output using clinical criteria. Hypoxic (ischemic) hepatitis $(\mathrm{HH})$ was said to be present if there was a clinical setting of circulatory failure, a sharp but transient increase in serum aminotransferase activities of at least 20 times the upper limit of normal, and after exclusion of other causes of hepatic necrosis. Of the 91 patients, 20 developed $\mathrm{HH}(2.6 \%$ of admissions to the coronary care unit; $21.9 \%$ of patients with low cardiac output), 48 did not develop $\mathrm{HH}$, and 23 died within 24 hours. Hepatic blood flow was measured in the 20 patients with $\mathrm{HH}$, and in 41 of the 48 surviving patients without $\mathrm{HH}$, using the method of galactose clearance at low concentrations. When compared with patients who did not develop $\mathrm{HH}$, patients with $\mathrm{HH}$ had lower hepatic blood flow $(867 \pm 377 \mathrm{ml} / \mathrm{min}$ vs 1429 $\pm 644 \mathrm{ml} / \mathrm{min})$, higher central venous pressure $\left(22.5 \mathrm{cmH}_{2} \mathrm{O}\right.$ vs $\left.14 \mathrm{cmH}_{2} \mathrm{O}\right)$, and a trend toward lower cardiac output (3.4 L/min vs $4.4 \mathrm{~L} / \mathrm{min}$ ). Interestingly, only approximately $50 \%$ of patients in each group had a systolic arterial pressure below $90 \mathrm{mmHg}$. The overall mortality was high $-55 \%$ in the $\mathrm{HH}$ group and $46 \%$ in the group without $\mathrm{HH}$.

\section{Citation count 26}

\section{Related references}

1. Gibson P, Dudley F. Ischemic hepatitis: clinical features, diagnosis and prognosis. Aust NZ J Med 1984; 14: 822-825. 
2. Fuchs S, Bogomolski-Yahalom V, Paltiel O, Ackerman Z. Ischemic hepatitis: clinical and laboratory observations of 34 patients. J Clin Gastroenterol 1998; 26: 183-186.

3. Garland J, Werlin S, Rice T. Ischemic hepatitis in children: diagnosis and clinical course. Crit Care Med 1988; 16: 1209-1212.

\section{Key message}

The findings of this study show that ischemic hepatitis is common in environments where there are patients with low cardiac output states, such as Coronary Care Units and Intensive Care Units. The classic hemodynamic pattern is low cardiac output (resulting in reduced hepatic blood flow), and high right atrial pressure. Hypotension is not a prerequisite. The mortality rate is $>50 \%$

\section{Why it's important}

This is the first prospective study to determine the incidence of ischemic hepatitis in patients with severe cardiac disease and low cardiac output. It is also by far the most comprehensive study of hemodynamic measurements in patients with ischemic hepatitis, and the only one to measure liver blood flow.

\section{Strengths}

This is a large, prospective, observational study of 766 patients admitted to a single intensive care unit over a 1-year period.

\section{Weaknesses}

Exclusion of the 23 patients who died within 24 hours of the episode of low cardiac output, before measurements of hepatic blood flow had been made, may have altered the findings, particularly regarding the incidence of ischemic hepatitis. The validity of the hepatic blood flow measurements themselves are open to some dispute - firstly because of the use of galactose clearance rather than indocyanine green clearance, and secondly because they were performed subsequent to the episode of low cardiac output, and therefore may not reflect the hemodynamic state at that time.

\section{Relevance}

Ischemic hepatitis occurs in up to 5\% of admissions to Intensive Care Units and Coronary Care Units, and is consequently more common than a number of other conditions that have attracted massive research interest. This paper describes the clinical setting, hemodynamic characteristics, and outcome of ischemic hepatitis, and consequently makes a major contribution to the body of knowledge on the subject. Ischemic hepatitis is not a rare hepatic disorder, but rather it is a circulatory disorder, and therefore of great relevance to intensive care medicine. 
Title

\section{Monoethylglycinexylidide formation kinetics: a novel approach to assessment of liver function}

\section{Author}

Oellerich M, Raude E, Burdelski M, Schulz M, Schmidt FW, Ringe B, Lamesch P, Pichlmayr R, Raith H, Scheruhn M, Wrenger M, Wittekind C

\section{Reference}

J Clin Chem Clin Biochem 1987; 25: 845-853

\section{Abstract}

A novel quantitative liver function test is described which is based on monoethylglycinexylidide (MEGX) formation after lidocaine bolus injection. Following the administration of small single doses of lidocaine hydrochloride $(1 \mathrm{mg} / \mathrm{kg})$, monoethylglycinexylidide serum concentration-time curves were determined by a novel, highly sensitive fluorescence polarization immunoassay (FPIA) in healthy volunteers, liver donors, and patients with liver cirrhosis. The FPIA allowed rapid and reliable monoethylglycinexylidide determinations in serum and urine (between-days coefficient of variation: < 10.3\%, recovery: $80-113 \%$ ). Monoethylglycinexylidide concentrations measured by FPIA in 32 serum samples from patients correlated well with those determined by HPLC. The monoethylglycinexylidide concentration in serum determined 15 minute after a lidocaine bolus injection proved to be a highly sensitive and specific indicator of hepatic dysfunction. Average monoethylglycinexylidide concentrations in serum obtained 15 minute after lidocaine injection were substantially lower in patients with liver cirrhosis than in healthy volunteers. The average monoethylglycinexylidide concentrations in serum were also substantially lower in liver donors with ballooning or fatty changes of hepatocytes than in donors without relevant alterations of liver histology. By means of monoethylglycinexylidide formation in the liver donors, primary function of the transplanted liver was correctly predicted in 32/37 cases, and initial non-function in $4 / 6$ cases.

\section{Summary}

Monoethylglycinexylidide (MEGX) is the first metabolite of lignocaine formed by oxidative de-ethylation by the hepatic cytochrome P-450 system. In this study, the appearance of MEGX measured by a fluorescence polarization immunoassay after a test dose of intravenous lignocaine was investigated as a liver function test. The findings showed that there was a satisfactory correlation between the measurement of MEGX by the novel fluorescence polarization immunoassay and by high performance liquid chromatography. The formation and elimination kinetics of MEGX were investigated for healthy volunteers, patients with histologically confirmed liver cirrhosis, and liver donors after a single intravenous bolus injection of lignocaine $(1 \mathrm{mg} / \mathrm{kg})$. In healthy volunteers, the peak MEGX concentration occurred 15 minutes after the bolus of lignocaine, whereas in patients with cirrhosis, its rate of formation was markedly decreased, and maximum concentrations were observed at about 4 hours. In liver donors the 15-minute MEGX concentration was lower in donors, with altered liver histology than in those with normal histology. Patients with cirrhosis had the lowest 15-minute MEGX concentration of all the groups. 


\section{Related references}

1. Oellerich M, Armstrong VW. The MEGX test: a tool for the real-time assessment of hepatic function. Ther Drug Monit 2001; 23: 81-92.

2. Maynard ND, Bihari DJ, Dalton RN et al. Liver function and splanchnic ischemia in critically ill patients. Chest 1997; 111: 180-187.

3. Schroter J, Wandel $\mathrm{C}$, Bohrer $\mathrm{H}$ et al. Lignocaine metabolite formation: an indicator for liver dysfunction and predictor of survival in surgical intensive care patients. Anesthesia 1995; 50: 850-854.

\section{Key message}

Conventional 'liver function tests' measure liver damage rather than liver function. The MEGX test assesses the liver's capacity to metabolize drugs (in this case lignocaine), and thus is a true 'liver function test'. The data presented in this study suggest that the 15-minute MEGX concentration varies with the adequacy of liver function.

\section{Why it's important}

This paper is the first to describe and assess the formation of MEGX after a standard dose of lignocaine as a dynamic liver function test.

\section{Strengths}

Novel approach.

\section{Weaknesses}

The test is not well validated in this report.

\section{Relevance}

Because of its ease of use and rapid turnaround, the MEGX test has found widespread application for the real-time assessment of hepatic function. In liver transplantation, the MEGX test has found a place in selection of transplant candidates, and in monitoring of liver recipients early after transplantation. In intensive care patients, a rapid decrease in MEGX test values is associated with increased risk of developing multiple organ failure, and a poor outcome, and consequently may have a role in investigation of the role of the liver in the multiple organ failure syndrome. 
Title

\section{Early indicators of prognosis in fulminant hepatic failure}

Author

O’Grady J, Alexander G, Hayllar K, Williams R

Reference

Gastroenterology 1989; 97: 439-445

\section{Abstract}

The successful use of orthotopic liver transplantation in fulminant hepatic failure has created a need for early prognostic indicators to select the patients most likely to benefit at a time when liver transplantation is still feasible. Univariate and multivariate analysis was performed on 588 patients with acute liver failure managed medically during 1973-1985, to identify the factors most likely to indicate a poor prognosis. In acetaminophen-induced fulminant hepatic failure, survival correlated with arterial blood $\mathrm{pH}$, peak prothrombin time, and serum creatinine $-\mathrm{a} \mathrm{pH}<7.30$, prothrombin time $>100$ seconds, and creatinine $>$ $300 \mu \mathrm{mol} / \mathrm{L}$ indicated a poor prognosis. In patients with viral hepatitis and drug reactions, three static variables [etiology (non A, non $B$ hepatitis or drug reactions), age $<11$ and $>40$ year, duration of jaundice before the onset of encephalopathy $>7$ days], and two dynamic variables (serum bilirubin $>300 \mu \mathrm{mol} / \mathrm{L}$, and prothrombin time $>50$ seconds) indicated a poor prognosis. The value of these indicators in determining outcome was tested retrospectively in a further 175 patients admitted during 1986-1987, leading to the construction of models for the selection of patients for liver transplantation.

\section{Summary}

The records of 588 patients with fulminant hepatic failure (FHF) and grade III or IV encephalopathy admitted to King's College Hospital, London, between 1973 and 1985, were scrutinized to identify simple parameters that might prove to be prognostic indicators. These parameters were examined by univariate and multivariate analysis to determine the factors most likely to be associated with poor prognosis. The analysis was performed in two groups, paracetamol-induced and non-paracetamol-induced, because of the higher incidence of renal failure and metabolic acidosis in the former group.

The prognostic indicators so determined were then examined retrospectively, in a second group of 175 patients with FHF admitted during 1986-1987, to determine their sensitivity and specificity in prediction of a fatal outcome. Models were then constructed for selection of patients for liver transplantation on the basis of an extremely poor prognosis with medical management.

The criteria adopted for paracetamol-induced FHF were $\mathrm{pH}<7.30$ (irrespective of grade of encephalopathy), or prothrombin time $>100$ seconds and serum creatinine $>300$ $\mu \mathrm{mol} / \mathrm{L}$ in patients with grade III or IV encephalopathy.

For FHF unrelated to paracetamol, the criteria determined were prothrombin time $>100$ seconds (irrespective of grade of encephalopathy), or any three of (irrespective of grade of encephalopathy): age $<10$ or $>40$ years, etiology (non-A, non-B hepatitis or idiosyncratic drug reactions), duration of jaundice before onset of encephalopathy $>7$ days, prothrombin time $>50$ seconds, and serum bilirubin $>300 \mu \mathrm{mol} / \mathrm{L}$. 


\section{Related references}

1. Tygstrup N, Ranek L. Assessment of prognosis in fulminant hepatic failure. Semin Liver Dis 1986; 6: 129-137.

2. Bernuau J, Goudeau A, Poynard T et al. Multivariate analysis of prognostic factors in fulminant hepatitis B. Hepatology 1986; 6: 648-651.

3. Anand AC, Nightingale P, Neuberger JM. Early indicators of prognosis in fulminant hepatic failure: and assessment of the King's criteria. J Hepatol 1997; 26: 62-68.

\section{Key message}

This study has developed criteria that predict death in patients with FHF who are managed medically. In the era of liver transplantation, these criteria are also used to select patients for liver transplantation. All patients with FHF should be discussed with a transplant unit. Patients who meet these criteria should be transplanted.

\section{Why it's important}

This paper has changed practice. It allows decisions to be made about transplantation in FHF so that unnecessary transplantation can be minimized. Because the criteria involve simple, easy to apply parameters that are readily available, the algorithm also permits decisions to transplant to be taken early, maximizing the period of time available to find a suitable donor, and allowing transplantation to be undertaken before the development of cerebral edema.

\section{Strengths}

This study is based on the largest number of patients in a single unit ever reported.

\section{Weaknesses}

Data were collected over a period of 13 years, and it is possible that one or more aspects of management may have changed in that time, and may independently affect outcome.

\section{Relevance}

Various models have been used to predict outcome in FHF, but the 'King's criteria' identified in this study are the most widely used. These criteria are based on the study of a huge number of patients treated medically, and, because so many patients are now transplanted, it is extremely unlikely that the study could ever be repeated. Consequently, the criteria identified in this paper are likely to be used widely to select transplant candidates for the foreseeable future. 
Title

\section{Emergency liver transplantation for fulminant hepatitis}

Author

Bismuth H, Samuel D, Gugenheim J, Castaing D, Bernuau J, Rueff B, Benhamou JP

\section{Reference}

Ann Intern Med 1987; 107: 337-341

\section{Abstract}

Orthotopic liver transplantation was done in 17 patients with fulminant hepatitis. The cause of the liver disease was infection with hepatitis B virus, or co-infection with hepatitis $B$ virus and hepatitis $D$ virus, or infection with hepatitis A virus in 6 patients; drug hepatotoxicity in 5; and indeterminate in 6. Grafts from incompatible blood groups, steatotic grafts, or reduced-size grafts were used in 5, 4, and 4 patients, respectively. Of the 17 patients, 5 died: 2 of early liver failure due to the poor quality of the graft, 1 presumably of accidentally transmitted acute infection with the human immunodeficiency virus, and 2 of decerebration occurring during or immediately after surgery. The 12 other patients were alive 2 to 15 months after transplantation.

\section{Summary}

This paper reports the clinical details and outcome of 17 patients with acute liver failure who underwent orthotopic liver transplantation in the mid-1980s, in the Hopital PaulBrousse, Villejuif, and the Hopital Beaujon, Clichy, France. Six patients had documented viral hepatitis, five had drug hepatotoxicity, and the cause was undetermined in the remaining six. Despite compromises necessitated by emergency transplantation (ABO and size incompatibility, and steatotic grafts), 12 patients survived. Deaths were due to cerebral edema (2), early graft failure (2), and graft-transmitted infection.

\section{Citation count 237}

\section{Related references}

1. Bismuth $H$, Samuel $D$, Castaing $D$, Williams R, Pereira SP. Liver transplantation in Europe for patients with acute liver failure. Semin Liver Dis 1996; 16: 415-425.

2. Sheil AGR, McCaughan GW, Isai H, Hawker F, Thompson JF, Dorney SFA. Acute and subfulminant liver failure: the role of liver transplantation. Med J Aust 1991; 154: 724-728.

3. Peleman RR, Gavaler JS, Van Thiel D et al. Orthotopic liver transplantation for acute and subacute hepatic failure in adults. Hepatology 1987; 3: 484-489.

\section{Key message}

Liver transplantation can be a successful treatment for acute liver failure, and, overall, the outcome with transplantation is better than with supportive medical management alone. 
Why it's important

This paper was the first large case study to document the feasibility and success of liver transplantation for acute liver failure.

\section{Strengths}

This is a succinct review of 17 patients with acute liver failure who underwent liver transplantation, with appropriate demographic, etiological, clinical, and outcome information that remains relevant many years later.

\section{Weaknesses}

No case control data are reported. Although it is stated that transplantation was undertaken for a rapidly deteriorating neurological condition and severe coagulation disorders, the outcome of patients who did not meet these criteria, or who met the criteria and could not be transplanted, is not reported. Hence, although this paper describes the feasibility of liver transplantation in this population, it does not assess the value.

\section{Relevance}

Liver transplantation, as described in this case series, remains the best chance of survival for the majority of patients with acute liver failure. Although criteria for transplantation, and aspects of the surgical technique and postoperative immunosuppression have developed over the intervening years, orthotopic liver transplantation has not been superceded, and should be considered as an option in all patients with acute liver failure. 
Title

\section{Improvement by acetylcysteine of hemodynamics and oxygen transport in fulminant hepatic failure}

Author

Harrison PM, Wendon JA, Gimson AES, Alexander GJM, Williams R

Reference

N Engl J Med 1991; 324: 1852-1857

Abstract

BACKGROUND. When administered early after an overdose of acetaminophen, intravenous acetylcysteine prevents hepatic necrosis by replenishing reduced stores of glutathione. How acetylcysteine improves the survival of patients with established liver damage induced by acetaminophen, however, is unknown. This study was undertaken to determine whether the beneficial effect of acetylcysteine under such circumstances could be due to enhancement of oxygen delivery and consumption. METHODS. We studied the effect of acetylcysteine on systemic hemodynamics and oxygen transport in 12 patients with acetaminophen-induced fulminant hepatic failure, and 8 patients with acute liver failure from other causes. The acetylcysteine was given in a dose of $150 \mathrm{mg} / \mathrm{kg}$ in $250 \mathrm{ml}$ of $5 \%$ dextrose over a period of 15 minutes, and then in a dose of $50 \mathrm{mg} / \mathrm{kg}$ in $500 \mathrm{ml}$ of $5 \%$ dextrose over a period of 4 hours; measurements were made before treatment began, and after 30 minutes of the regimen. RESULTS. In the patients with acetaminophen-induced liver failure, the infusion of acetylcysteine resulted in an increase in mean oxygen delivery from 856 to $975 \mathrm{ml} / \mathrm{min} / \mathrm{m}^{2}$ ( $\left.p=0.0036\right)$, due to an increase in the cardiac index from 5.6 to $6.7 \mathrm{~mm} / \mathrm{m}^{2}(p=0.0021)$. Mean arterial pressure rose from 88 to $95 \mathrm{mmHg}(p=0.0054)$, despite a decrease in systemic vascular resistance from 1296 to 1113 dyn.sec.cm-5 per square meter $(p=0.027)$. There was an increase in oxygen consumption from 127 to 184 $\mathrm{ml} / \mathrm{min} / \mathrm{m}^{2}(\mathrm{p}=0.0007)$ associated with an increase in the oxygen-extraction ratio from 16 to 21 percent $(p=0.022)$. The effects in the patients with acute liver failure from other causes were similar. CONCLUSIONS. The increase in oxygen delivery and consumption in response to acetylcysteine may account for its beneficial effect on survival in patients with fulminant hepatic failure induced by acetaminophen.

\section{Summary}

The effects of intravenous n-acetylcysteine on systemic hemodynamics and oxygen transport were studied in 12 patients with paracetamol-induced acute liver failure, and eight patients with acute liver failure from other causes. Measurements were made before a bolus dose of $150 \mathrm{mg} / \mathrm{kg}$ of n-acetylcysteine given over 15 minutes, and 30 minutes after commencement of an infusion of $50 \mathrm{mg} / \mathrm{kg}$ of n-acetylcysteine given over 4 hours.

The findings were that in patients with paracetamol-induced acute liver failure, infusion of $n$-acetylcysteine resulted in an increase in mean oxygen delivery from 856 to 975 $\mathrm{ml} / \mathrm{min} / \mathrm{m}^{2}$, resulting mainly from an increase in the mean cardiac index by approximately $20 \%\left(5.6\right.$ to $6.7 \mathrm{~L} / \mathrm{min} / \mathrm{m}^{2}$ ). Mean arterial pressure increased from 88 to $95 \mathrm{mmHg}$ despite a decrease in the mean systemic vascular resistance from 1296 to $1113 \mathrm{dyn} / \mathrm{sec} / \mathrm{cm}^{-5}$. In patients with acute liver failure from other causes, the effects of n-acetylcysteine were similar but less marked, and there were significant changes (increases) only for oxygen consumption and oxygen extraction ratio. 
Each patient had similar measurements performed before and during an infusion of prostacyclin at a rate of $5 \mathrm{ng} / \mathrm{kg}$, and again when $\mathrm{n}$-acetylcysteine and prostacyclin were infused simultaneously. The effects of prostacyclin were similar to those of n-acetylcysteine, but there was a decrease in mean arterial pressure rather than an increase, and a much smaller increase in oxygen extraction.

Four patients with acute liver failure secondary to paracetamol were studied after recovery. In this group, n-acetylcysteine had no effect on any hemodynamic or oxygen transport variable. However, with infusion of prostacyclin, there was an increase in cardiac index, decrease in systemic vascular resistance, and increased oxygen delivery without an increase in consumption.

\section{Citation count 255}

\section{Related references}

1. Walsh TS, Hopton P, Philips BJ, Mackenzie SJ, Lee A. The effect of n-acetylcysteine on oxygen transport and uptake in patients with fulminant hepatic failure. Hepatology 1998; 27: 1332-1340.

2. Harrison PM, Keays R, Bray GP, Alexander GJ, Williams R. Improved outcome of paracetamol-induced fulminant hepatic failure by late administration of acetylcysteine. Lancet 1990; 335: 1572-1573.

3. Rank R, Michel C, Haertel C et al. N-acetylcysteine increases liver blood flow and improves liver function in septic shock patients: results of a prospective, randomised, double-blind study. Crit Care Med 2000; 28: 3799-3807.

\section{Key message}

There is a characteristic high output-low resistance hemodynamic state in fulminant hepatic failure that may be associated with covert tissue hypoxia. Intravenous infusion of n-acetylcysteine was associated with an increase in oxygen delivery and consumption, and also in tissue oxygen extraction in patients with fulminant hepatic failure. It is postulated that this may account for the beneficial effect of n-acetylcysteine on survival in patients with acute liver failure caused by paracetamol, and further, may be of benefit in patients with multiple organ failure from other causes, such as sepsis.

\section{Why it's important}

This study is the justification for the widespread use of n-acetylcysteine in patients with acute liver failure, and it has also provoked investigation of possible clinical benefits in other conditions, such as septic shock.

\section{Strengths}

This is a comprehensive clinical study of a relatively homogeneous group of patients.

\section{Weaknesses}

Because the Fick method was used to calculate oxygen consumption from the cardiac output and the arteriovenous oxygen content difference, it is possible that an artifactual relationship between oxygen delivery and consumption was shown because of mathematical coupling. It is now generally accepted that oxygen delivery and consumption should be determined independently. 


\section{Relevance}

Although undoubtedly a classic study, the findings presented in this paper have not been confirmed in a similar more recent study (see Related reference no. 1). In the latter study, oxygen consumption was measured independently of oxygen delivery, using a method based on respiratory gas analysis, and patients were studied over a longer period. Nevertheless, the paper under review has been responsible in part for a resurgence of interest in n-acetylcysteine as a therapeutic agent, not only in patients with acute liver failure, but also in other groups of critically ill patients, such as those with sepsis, acute lung injury, and multiple organ failure. As with acute hepatic failure, published studies of other groups of critically ill patients have shown inconsistent findings, and the role of n-acetylcysteine in critically ill patients remains unclear. 
Title

\section{Acute hepatic coma treated by cross-circulation with a baboon and by repeated exchange transfusions}

Author

Saunders SJ, Terblanche J, Bosman SC, Harrison GG, Walls R, Hickman R, BieBuyck J, Dent D, Pearce S, Barnard C

\section{Reference}

Lancet 1968; 2: 585-588

Abstract

Not available

\section{Summary}

The case is reported of a 29-year-old woman who developed presumptive viral hepatitis. Her jaundice initially resolved after 6 weeks, but recurred soon after and was accompanied by a flapping tremor. Her neurological state deteriorated markedly to grade IV encephalopathy. She was treated with a regimen of daily exchange transfusion for 6 days, but despite some apparent improvement related to these procedures, this was not sustained. She duly became apneic, and after an unsuccessful seventh exchange transfusion, she underwent cross-circulation with a baboon. The baboon had been especially prepared by washing out the blood volume with lactate solution under hypothermia on bypass, and then replacing the blood volume with human blood compatible with the patient. After about 12 hours, spontaneous ventilation returned, and there was an improvement in conscious state and the treatment was terminated. She remained neurologically impaired for two further days, and then improved markedly to become fully conscious with no abnormal neurological signs. Her improvement persisted until publication of the case report.

\section{Citation count 52}

\section{Related references}

1. Stange J, Mitzner SR, Risler T et al. Molecular adsorbent recycling system (MARS): clinical results of a new-membrane-based blood purification system for bioartificial liver support. Artif Organs 1999; 23: 319-330.

2. Sussman NL, Kelly JH. Extracorporeal liver support: cell-based therapy for the failing liver. Am J Kidney Dis 1997; 30 (5 Suppl 4): S66-S71.

3. Detry O, Arkadopoulos N, Ting P et al. Clinical use of a bioartificial liver in the treatment of acetominophen-induced fulminant hepatic failure. Am Surg 1999; 65: 934-938.

\section{Key message}

Complex forms of extracorporeal perfusion are possible in critically ill patients with acute liver failure and advanced encephalopathy. A focus on metabolic support (for example from functioning hepatocytes) rather than blood purification will most likely be successful. 


\section{Why it's important}

This paper marks the beginning of a long and still-continuing struggle to find a clinically relevant artificial liver device that can be used as a bridge to regeneration or transplantation in patients with acute liver failure.

\section{Strengths}

This is a report of original and far-sighted ideas that must have stretched the boundaries of knowledge and technology in the late 1960s.

\section{Weaknesses}

This is a case report only. It is possible that the improvement after cross-circulation may have been incidental, and the patient may have recovered spontaneously without it.

\section{Relevance}

The precise technique used in this report is most unlikely to be used again because of concerns about transmission of infection, and immunological considerations. However, more than 30 years later, there remains a need to develop a safe and effective artificial liver for use in patients with acute liver failure. 
Title

\section{Effect of intravenous albumin on renal impairment and mortality in patients with cirrhosis and spontaneous bacterial peritonitis}

Author

Sort P, Navasa M, Arroyo V, Aldeguer X, Planas R, Ruiz-Del-Arbol L, Castells L, Vargas V, Soriano G, Guevara M, Gines P, Rodes J

\section{Reference}

N Engl J Med 1999; 341: 403-409

\section{Abstract}

BACKGROUND: In patients with cirrhosis and spontaneous bacterial peritonitis, renal function frequently becomes impaired. This impairment is probably related to a reduction in effective arterial blood volume, and is associated with a high mortality rate. We conducted a study to determine whether plasma volume expansion with intravenous albumin prevents renal impairment and reduces mortality in these patients. METHODS: We randomly assigned 126 patients with cirrhosis and spontaneous bacterial peritonitis to treatment with intravenous cefotaxime (63 patients), or cefotaxime and intravenous albumin (63 patients). Cefotaxime was given daily in dosages that varied according to the serum creatinine level, and albumin was given at a dose of $1.5 \mathrm{~kg}$ of body weight at the time of diagnosis, followed by $1 \mathrm{~kg}$ on day 3 . Renal impairment was defined as nonreversible deterioration of renal function during hospitalization. RESULTS: The infection resolved in 59 patients in the cefotaxime group (94\%) and 62 in the cefotaxime-plus-albumin group $(98 \%)(p=0.36)$. Renal impairment developed in 21 patients in the cefotaxime group $(33 \%)$, and 6 in the cefotaxime-plus-albumin group (10\%) $(p=0.002)$. Eighteen patients $(29 \%)$ in the cefotaxime group died in the hospital, as compared with 6 (10 percent) in the cefotaxime-plus-albumin group ( $p=0.01)$; at three months, the mortality rates were 41 percent (a total of 26 deaths), and $22 \%$ (a total of 14 deaths), respectively ( $p=0.03$ ). Patients treated with cefotaxime had higher levels of plasma renin activity than those treated with cefotaxime and albumin; patients with renal impairment had the highest values. CONCLUSIONS: In patients with cirrhosis and spontaneous bacterial peritonitis, treatment with intravenous albumin in addition to an antibiotic reduces the incidence of renal impairment and death, in comparison with treatment with an antibiotic alone.

\section{Summary}

The effect of intravenous albumin on renal impairment and mortality was studied in 126 patients with cirrhosis and spontaneous bacterial peritonitis. Patients were randomly assigned to receive treatment with intravenous cefotaxime or cefotaxime and intravenous albumin. Albumin was given at a dose of $1.5 \mathrm{~g} / \mathrm{kg}$ body weight at the time of diagnosis, and $1 \mathrm{~g} / \mathrm{kg}$ on day 3 .

The infection resolved in a similar number of patients in both groups. However, the incidence of renal impairment was very much lower in the cefotaxime plus albumin group (6 patients; 10\%) than in the cefotaxime alone group (21 patients; 33\%). Furthermore, the mortality rates were lower in the cefotaxime plus albumin groups, i.e. 6 patients or $10 \%$ vs 18 patients or $29 \%$ for in-hospital mortality, and 14 patients (22\%) and 26 patients (41\%) at 3 months. 
Patients treated with cefotaxime had higher levels of plasma renin activity than those treated with cefotaxime and albumin, and patients with renal impairment had the highest values.

The authors conclude that in patients with cirrhosis and spontaneous bacterial peritonitis, treatment with intravenous albumin in addition to an antibiotic reduces the incidence of renal impairment and death in comparison with an antibiotic alone.

\section{Citation count 262}

\section{Related references}

1. Guarner C, Soriano G. Spontaneous bacterial peritonitis. Semin Liver Dis 1997; 17: 203-217.

2. Navasa M, Rimola A, Rodes J. Bacterial infections in liver disease. Semin Liver Dis 1997; 17: 323-333.

3. Arroyo V, Gines P, Gerbes AL et al. Definition and diagnostic criteria of refractory ascites and hepatorenal syndrome in cirrhosis. International Ascites Club. Hepatology 1996; 23: 164-176.

Key message

Spontaneous bacterial peritonitis is a common complication in patients with cirrhosis and ascites, and may be accompanied by renal impairment, development or worsening of encephalopathy, and increased risk of death. Treatment with non-nephrotoxic antibiotics has been associated with improved outcome, but volume expansion with albumin in addition to antibiotics results in further improvement in survival and a reduced incidence of renal impairment. The most likely explanation is that albumin infusion prevents hypovolemia and the subsequent activation of vasoconstrictor systems. However, mechanisms unrelated to plasma expansion cannot be ruled out.

Why it's important

This study provides strong evidence that intravenous albumin therapy should be given, in addition to antibiotic therapy, to patients with cirrhosis and spontaneous bacterial peritonitis. The effects of albumin therapy are not known in patients with organic nephropathy and serum creatinine concentration $>265 \mu \mathrm{mol} / \mathrm{L}$ because these groups were excluded from the study.

Strengths

This is a well-designed, randomized, controlled trial from the group who virtually singlehandedly have defined the management of ascites in cirrhosis.

\section{Weaknesses}

There are few. Further studies should investigate the effect of different doses of albumin, and whether other plasma expanders are equally effective.

Relevance

This study does not specifically involve patients treated in an intensive care unit. Nevertheless, spontaneous bacterial peritonitis is responsible for many admissions of cirrhotic patients to the ICU, as it is sometimes a silent precipitant of gastrointestinal bleeding, encephalopathy, and renal impairment, as well as overt sepsis. Indeed, it should be excluded in every cirrhotic patient admitted to the ICU. Consequently, advances in its treatment are very relevant to the ICU clinician. Furthermore, despite the current uncertainty as to the place of albumin therapy in critically ill patients, it seems clear that it confers a survival advantage to patients with cirrhosis and spontaneous bacterial peritonitis. 